\title{
Carnets
}

Revue électronique d'études françaises de l'APEF

Deuxième série - 9 | 2017

Reconnaissances et légitimité en français

\section{Jean-Marie Gustave Le Clézio et Patrick Modiano dans le champ intellectuel européen}

\section{Pilar Andrade Boué}

\section{(2) OpenEdition}

Journals

Édition électronique

URL : http://journals.openedition.org/carnets/2052

DOI : 10.4000/carnets.2052

ISSN : 1646-7698

Éditeur

APEF

Référence électronique

Pilar Andrade Boué, « Jean-Marie Gustave Le Clézio et Patrick Modiano dans le champ intellectuel européen », Carnets [En ligne], Deuxième série - 9 | 2017, mis en ligne le 31 janvier 2017, consulté le 10 décembre 2020. URL : http://journals.openedition.org/carnets/2052 ; DOI : https://doi.org/10.4000/ carnets. 2052

Ce document a été généré automatiquement le 10 décembre 2020.

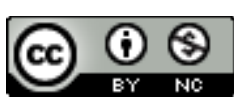

Carnets est mis à disposition selon les termes de la licence Creative Commons - Atribution - Pas d'utilisation commerciale 4.0 International. 


\title{
Jean-Marie Gustave Le Clézio et Patrick Modiano dans le champ intellectuel européen
}

\author{
Pilar Andrade Boué
}

1 Six ans séparent les deux prix Nobels décernés à des écrivains de langue française : Le Clézio en 2008, Modiano en 2014. Même si la France est le pays possédant le plus de lauréats au monde ${ }^{1}$, ceci est un fait plutôt rare, dans un monde aussi ample et abondant en livres que le nôtre. Mais nous ne pouvons, personnellement, que nous en réjouir, car cela nous a permis d'élaborer ce travail, où nous proposons d'examiner la situation de ces deux auteurs dans la République des lettres et leurs pas vers la consécration définitive. Nous savons en effet que le Nobel légitime et promeut amplement un auteur au sein du champ littéraire européen, et le consacre dans le champ littéraire français. Désormais, il aura une postérité ; il figurera dans les bibliothèques les plus prestigieuses du continent, et dans les manuels scolaires des bacheliers francophones. « Un Nobel de littérature, quel que soit le regard détaché que l'on porte sur tout hochet, exerce une fonction fédératrice. Il rassemble des lecteurs de tous horizons autour d'une œuvre et [crée un] effet tout littéraire, tout laïc, de communion ", ainsi que l'exprime Blanckeman $(2010: 265)^{2}$.

2 Nous allons donc nous pencher ici sur les éléments qui ont contribué au succès de ces deux auteurs, autres bien sûr que le plus évident de tous, celui de la qualité intrinsèque de leurs textes. De ces éléments non essentiels nous nommerons notamment: 1) le capital familial et social de départ des auteurs, 2) les sujets abordés dans leurs œuvres, en tant qu'accélérateurs ou décélérateurs du cursus honorum, et 3) les stratégies promotionnelles et médiatiques de la réussite.

3 L'analyse de ces trois aspects nous aidera à comprendre quels ont été les éléments de légitimation préexistants et les mécanismes de légitimation mis en œuvre aussi bien par Le Clézio et Modiano eux-mêmes que par le marché et les maisons d'édition, pour positionner ces deux auteurs dans le champ intellectuel européen. Champ où, ainsi que Pascale Casanova l'avait expliqué (1999, passim), le fait d'appartenir à la culture française 
et d'écrire en français est un atout très important, puisqu'il s'agit déjà d'un facteur de prestige.

\section{Capital familial et social de départ}

4 Commençons donc par l'étude du capital familial et social de Le Clézio et de Modiano, c'est-à-dire des aspects de leur biographie qui les ont aidés à faire partie du champ intellectuel français. Il convient tout d'abord de souligner la différence importante qui sépare nos auteurs en ce qui concerne leur origine et leur évolution au sein d'un groupe social.

Le Clézio appartient à une famille française, émigrée à l'île Maurice puis expulsée. Il a épousé Rosalie Piquemal, polonaise, et en deuxième noce Jémia Jean, d'origine marocaine - deux femmes inconnues dans le monde; il habite au Mexique, au pied d'un volcan, et il voyage souvent à Paris, à Nice ou à Maurice (Cortanze, 2009 : 36).

6 Modiano, pour sa part, provient d'une famille juive, on le sait déjà, installée d'abord en Italie, ensuite en Grèce, puis en France. Son père, pendant l'Occupation, s'entoure d'affairistes, de collaborateurs, de personnes recherchées par la justice (Cosnard, 2010: 17); sa mère fréquente des gens du théâtre et du cinéma (Cosnard, 2010: 115-117). Modiano enfant est envoyé dans des écoles de prestige, telles le collège de Montcel à Jouyen-Josas (octobre 1956-été 1960), où s'est installée les dernières années la fondation Cartier, le collège-lycée Saint-Joseph, en Haute-Savoie (septembre 1960-1962), et le Lycée Henri IV à Paris (septembre 1962-été 1964) 3. Là, l'écrivain a certainement commencé à fréquenter des milieux bourgeois, qui lui ont permis de connaître Denise Zehrfuss (rencontrée dans un restaurant des Champs Elysées), puis de l'épouser. Rappelons encore que les témoins de ce mariage ont été André Malraux, ami des Zehrfuss, et Raymond Queneau, ancien professeur et ami de Modiano. Quant à la fiancée, de mère juive, c'est la fille de Bernard Zehrfuss, célèbre architecte fonctionnaliste, figure majeure de l'architecture des Trente Glorieuses et premier Grand Prix de Rome en 1939, membre de l'Institut et secrétaire perpétuel de l'Académie des Beaux-Arts. Modiano, s'adaptant à ce milieu-là, a fait le choix d'habiter place Saint-Sulpice, dans le très coté $\mathrm{VI}^{\mathrm{e}}$ arrondissement. Ces éléments aident également à dessiner le profil socioculturel du personnage, et à estimer son potentiel dans le champ culturel.

7 En ce qui concerne la situation dans les milieux universitaires, en tant qu'étudiants et, plus tard, en tant que professeurs, rappelons que Modiano n'a pas fait d'études supérieures, qu'il avait associées probablement à une autorité paternelle vécue comme une tyrannie. En effet, son père l'avait inscrit en hypokhâgne au lycée Michel de Montaigne de Bordeaux pour l'éloigner de Paris, sur les instances de la belle-mère du jeune Patrick. Le futur écrivain s'enfuit de cette institution et, un an plus tard (1965) s'inscrivit à la Faculté des Lettres de la Sorbonne pour prolonger son sursis militaire ${ }^{4}$, un peu comme aujourd'hui on s'inscrit (à Madrid du moins) pour obtenir une bourse ou le ticket de transport moins cher. L'éloignement des instances universitaires est donc complet, ce qui est certainement un handicap pour sa promotion médiatique, handicap tempéré seulement par son côté moitié enfant terrible, moitié mauvais garçon, qui lui donnent un bonus.

8 Le Clézio, quant à lui, a fait des études à l'Université de Bristol et à Nice, puis a soutenu en 1964 un travail de fin d'études sur Henri Michaux et en 1977 une thèse d'histoire 
(traduction et présentation d'un texte du $\mathrm{XvI}^{\mathrm{e}}$ siècle) à l'Institut d'études mexicaines de Perpignan (Cortanze, 1999: 176). En outre, il a été enseignant à l'Université d'Albuquerque, au Mexique et, en 2003, il a occupé une chaire de professeur invité à l'université d'Ewha, à Séoul, en Corée, pays dont il a étudié l'histoire, la mythologie et les rites chamaniques, tout en enseignant la poésie et le roman français (Cortanze, 2009: 132). Notons enfin qu'il fut refusé au C.N.R.S., où il s'était porté candidat à deux reprises en 1978 et 1979 (Cortanze, 1999 : 179).

Ces balises biographiques nous permettent d'affirmer que Le Clézio a un capital familial et social très faible, mais un solide capital scolaire (formation reçue et donnée au sein d'institutions officielles), à l'inverse de Modiano, dont le capital scolaire est faible mais le capital familial et surtout social est très solide. C'est d'ailleurs ce dernier qui lui permit de tenter sa chance dans le monde de la chanson, autour de 1966, et dans le monde du théâtre, en 1974 ; la première tentative finira tôt et la deuxième encore plus tôt car, selon l'auteur, «on ne m'a pas laissé le temps » (Cosnard, $2010: 114)$. Enfin, la carrière de la mère lui permettra de mener à bien les projets cinématographiques que l'on connaît (Lacombe Lucien, Un innocent, etc.) parmi d'autres non réussis.

\section{Thématique et champ littéraire}

Il est vrai que les romanciers sont de moins en moins lus, et de plus en plus vus. Donc si nous parlons ici des sujets des œuvres, c'est juste sur l'hypothèse que ces sujets sont connus du public - à défaut d'avoir été lus ${ }^{5}$.

11 Examinons d'abord la thématique leclézienne ${ }^{6}$. Le prix Nobel définit l'auteur de Désert comme un « écrivain de nouveaux départs, de l'aventure poétique et de l'extase sensuelle, explorateur d'une humanité au-delà et en dessous de la civilisation régnante » (Le Clézio, 2008). Ces sujets sont en principe parfaitement susceptibles de légitimation, mais notons que, dans la dernière expression, perce de l'inconformisme (« d'une humanité au-delà et en dessous de la civilisation régnante ») qui était apparu très tôt dans la biographie leclézienne, lorsque, pendant son service militaire, le jeune Jean-Marie dénonça la prostitution en Thaillande ${ }^{7}$. Cet esprit de justicier deviendra, avec le temps, contestation permanente du système de vie occidental : « Maudite race blanche, dont je suis, et qui ne veut rien changer» (Cortanze, 2009: 50). La contestation, politiquement incorrecte, a-telle fait reculer le nom de Le Clézio dans la hiérarchie du champ littéraire ? Il se pourrait, au contraire, qu'elle ait fait augmenter sa popularité, car elle a contribué -probablement malgré lui- à la mise en scène médiatique du personnage. Nous retrouvons ce paradoxe médiatique lorsque l'écrivain aborde certains sujets. Par exemple, lors de son éloge de la civilisation aztèque avant la conquête espagnole, il a été traité de «barbare païen » et d'apologiste du "fascisme aztèque » par Guy Scarpetta dans la revue Globe, et lorsqu'il a publié une nouvelle dans la Revue d'études palestiniennes, Bernard-Henri Lévy l'a accusé d'être " un antisioniste déchaîné » (Garcin, 2008). Son prestige en a-t-il souffert ? Ou au contraire le tapage médiatique l'a, en réalité, propulsé vers le haut?

D'autres positions politiques et religieuses de Le Clézio choquent la doxa et, même si l'intention est pure, contribuent à maintenir l'auteur dans les ondes. Donnons-en deux exemples $^{8}$ : dans sa Lettre à ma fille du 12 janvier 2015, le Nobel s'est montré conciliateur envers les assassins de Charlie, qui, a-t-il dit, n'ont pas « été maîtres de leur destin » (Le Clézio, 2015a). Ces propos ont déclenché un tollé dans la presse islamophobe, qui l'a 
accusé de prôner «la collaboration en attendant la soumission»(Graven, 2015). Deuxième exemple : le 7 mars 2015, il a promis de rendre son passeport français si Marine Le Pen gagnait les élections, et a ajouté qu'il ne comprenait pas les Français. Cette opinion parue dans un petit journal argentin a été repêchée directement par les journaux à grand tirage de l'Hexagone, dont Le Point, où des lecteurs indignés ont vu en lui un francophobe et ont souhaité qu'il rende effectivement son passeport... et son compte bancaire (Le Clézio, 2015b).

13 C'est probablement ce penchant de polémiste qui dérange Modiano, très discret quand il s'agit de parler politique, et lui fait non seulement commenter le versant «moraliste » de Le Clézio, mais suggérer aussi que ce moralisme a aidé à l'attribution du prix Nobel :

Il était assez logique que Le Clézio le reçoive car tous les écrivains français lauréats, Romain Rolland, Anatole France, François Mauriac, s'inscrivent d'une certaine façon dans une tradition d'écrivains avec un arrière-fond... comment dire... un peu moraliste. Dans les autres pays, les primés sont plutôt des marginaux, comme Faulkner ou Hermann Hesse (Modiano 2010).

Pourtant, il est peut-être exagéré de suggérer, comme le fait la journaliste Nelly Kaprièlian, que Modiano se situe en marge des problèmes modernes, que sa littérature est essentielle, qu'elle «ne surfe ni sur les modes, ni sur les engouements politiques ». Kaprièlian parle, à propos de l'auteur de Dora Bruder, de l'« extraction de tout impératif sociétal (...), de toute doxa » (Kaprièlian, 2014)9. Il est vrai que Modiano a reconnu avoir une "espèce d'allergie, une méfiance instinctive de la politique et des hommes politiques» (Modiano, 2010), et que, par exemple, à la question "Que pensezvous de lui en tant que ministre de la Culture? », il s'est dérobé en disant : «Je ne pense rien du ministre de la Culture. De toute façon, à part Malraux, on les a tous oubliés. » (Modiano, 2010).

Toutefois, sans nier la réserve de Modiano, évidente notamment dans les médias, nous pensons que dans les entrelacs de sa prose on peut déceler un fort engagement. N'oublions pas que le roman que nous venons de citer, Dora Bruder, mettait en vedette un des tabous de l'historiographie officielle des Trente Glorieuses, la déportation de juifs français en Allemagne et leur mort dans des camps de concentration. N'oublions pas, non plus, que la Trilogie de l'occupation abordait aussi le mystère du collaborationnisme en France à une époque où personne n'osait en parler. Souvenons-nous également du scénario de Lacombe Lucien, le premier film traitant ce même sujet.

De plus, en marge de ces aspects, il y a une défense et illustration de la patrie chez Modiano, de grand-père cosmopolite et de père apatride. Dans Livret de famille, le héros rêve de s'établir en France (Modiano, 1977 : 157) et dans Villa Triste, d'épouser un "être exotique et presque inaccessible: une petite Française»(Modiano, 1975: 132). La cohésion familiale, des parents qui encadrent leurs enfants, sont des valeurs implicitement défendues a contrario dans les textes de Modiano; dans De si braves garçons s'énoncent les avantages de l'éducation de l'internat (les "bienfaits d'une discipline") et son rôle de foyer, à défaut d'un autre (le « réconfort d'une patrie », Modiano, 1982 : 9).

Ajoutons à ces opinions que, si l'on en croit le journaliste Jacques Chancel (2014, min. 3 : 08), notre auteur citait De Gaulle comme son personnage historique préféré (Cosnard, 2010 : 91), et qu'il se présentait, en 1972, comme un admirateur des styles de Paul Morand et de Louis-Ferdinand Céline, vichiste et antisémite respectivement notoires. Ce qui montre bien que Modiano place la qualité littéraire au-dessus des convictions idéologiques - ou alors qu'il se complaît dans l'ambigüité : rappelons encore que, lorsque 
La place de l'Étoile est publiée, ce sont des écrivains de droite et les « hussards » qui ont salué avec le plus d'enthousiasme cet écrivain rétro. Et dans son entretien avec Mitterrand sur le plateau d'Apostrophes (1978), le socialiste voit chez Modiano la figure Emmanuel Berl (juif de gauche qui a été interviewé par le futur Nobel), et Modiano admire un Mitterrand très... régionaliste! Plus tard toutefois, lors de la publication de L'horizon, Modiano a expliqué son goût pour Apollinaire et Maeterlinck (Cosnard, $2010: 7$ ).

À l'opposé, Le Clézio se réclame des présocratiques, de Lautréamont, d'Henri Michaux et de Francis Ponge; Lautréamont el Michaux font partie du personnel contestataire du champ littéraire français.

\section{Stratégies promotionnelles}

19 Régis Debray et d'autres médiologues nous ont appris qu'« on ne peut séparer une opération de pensée, à quelque époque que ce soit, des conditions techniques d'inscription, de transmission et de stockage qui la rendent possible» (Debray, 1991 : 229). Les écrivains doivent ainsi, comme nous professeurs des universités, travailler à leur visibilité, s'ils veulent intégrer la République des lettres. Aspect un peu délaissé par nos deux auteurs (surtout par Modiano) qui, dit-on, ne se sont pas prodigués dans les médias, et dont les indices de citations sont plus bas qu'il ne faudrait pour avoir un impact optimal dans le champ intellectuel.

Et pourtant les articles de journaux et les travaux académiques sur leurs œuvres foisonnent, ce qui est bien pour l'impact - comme à la fin du Moyen Âge, la glose drape le texte originel.

Quant à leur visibilité directe sur le net, les vidéos sur Youtube travaillant à la légitimité de ces écrivains sont assez nombreux. Mais Modiano, à cause de ses difficultés d'élocution, a préféré élaborer son image à travers la photo. Ainsi son corps est maintenant l'objet fétichisé d'un public indiscret avide de sensationnalisme et de sentimentalité. Deux livres contenant du matériel varié sur l'auteur ont été publiés. Le premier en 2012, dans la collection des Cahiers de l'Herne; on y trouve de multiples documents le concernant, tels que des certificats médicaux, des lettres d'amis, des petites notes des maitres, et bien sûr, des photos. Le deuxième livre est une édition des romans modianiens publié par Gallimard dans la collection Quarto, en 2013; il rassemble 62 illustrations montrant ses parents, son frère, son épouse, Queneau, Rubirosa, etc., dans le but d'insister sur le côté autobiographique du roman avec des décors et des figures réelles.

La première de couverture du volume des Cahiers de l'Herne montre un homme décontracté, habillé correctement, voire même un peu négligé, assis dans un fauteuil Louis XV. Cette attitude est reprise, accompagnée de deux tableaux inégaux et avec un air plus sérieux, sur la première de couverture du Quarto. Sur les deux photos, l'apparence de naturel cache une recherche approfondie qui vise à provoquer chez le récepteur une rêverie de l'identité. Bruno Blanckeman a étudié, à ce propos, deux autres photos de Modiano, expliquant que les poses s'adaptent aux goûts de l'époque. Mais Blanckeman va encore plus loin, suggérant aussi que le Nobel tente, à travers ses photos, de présenter son style et ses œuvres : « Entre un corps d'auteur ainsi saisi au bord de la syncope (...), et celui d'une œuvre écrite à même ses manques, le lien pourrait alors se préciser » (2003 : 
117). Donc les images seraient une réclame pour attirer les lecteurs en leur parlant des textes. Le « faire-lire » suivrait le « faire-voir ».

Comparons enfin cet étalage modianien avec celui de Le Clézio, toujours sérieux sur les photos, le regard souvent détourné de l'appareil. Les ouvrages biographiques de Cortanze reproduisent des images qui véhiculent l'idée de sobriété que Le Clézio a toujours appliqué à sa vie ; l'une d'elles est même l'exemple de photo peu avantageuse : l'écrivain a été pris de face, en contreplongée et vêtu d'un manteau noir qui occupe une grande partie du champ (Cortanze, 2009: 113). Il est clair que Le Clézio délaisse la séduction par le visage ou le corps - séduction pourtant bien facile dans son cas.

\subsection{Mécanismes institutionnels de légitimation : prix littéraires, participation à des jurys littéraires, publications dans des maisons d'édition prestigieuses} depuis les petits prix jusqu'au méga-prix Nobel (nous parlons pour ce dernier d'environ un million d'euros). En revanche, ils ont tous les deux refusé d'entrer à l'Académie française.

Si l'on compare les prix et distinctions reçus par les deux auteurs, on s'aperçoit d'abord que Modiano a reçu le Goncourt, alors que Le Clézio a dû se contenter du Renaudot, son pendant complémentaire. Rappelons que le montant du premier est de dix euros et le deuxième est honorifique, mais les récompenses pécuniaires s'exprimeront en chiffres de vente, et les récompenses en légitimité dans le champ littéraire sont énormes. Ils ont en outre reçu tous les deux le Prix Prince Pierre de Monaco et le prix Paul Morand, et ont été promus Officiers de la Légion d'Honneur. Rappelons que Modiano était déjà Chevalier (depuis 1996) avant même d'obtenir le Nobel.

Quant à la participation à des jurys littéraires, Le Clézio ne gagne toujours pas la manche : il a figuré au comité de lecture de Gallimard et au jury de la Cinéfondation et des courtsmétrages du Festival de Cannes, mais Modiano aussi.

Enfin, en ce qui concerne les maisons d'édition qui ont publié les œuvres des deux Nobels, c'est Gallimard qui a accaparé la presque totalité des parutions, à l'exception de quelques titres de Le Clézio parus chez Skira, Fata Morgana, Arléa et Le Seuil, et trois romans de Modiano confiés à Le Seuil entre 1988 et 1993. Mais pour le reste, Gallimard a monopolisé les ventes et augmenté le capital culturel symbolique des deux auteurs, étant donné le caractère « intellectuel» du public auquel s'adresse cette maison d'édition (Bourdieu, 1992 : 255). Ceci n'a pas empêché aux auteurs de critiquer les vices du marché éditorial. Ainsi Modiano soutient que les "maisons d'édition, (...) sont devenues des usines " (2010), et Le Clézio commente les « difficultés que les jeunes ont à se faire publier » et « la relativité du système éditorial $»^{10}$.

D'ailleurs, aucun des écrivains ne s'est trop plié aux exigences du marché, nous semble-t$\mathrm{il}$, malgré les contraintes subies par tout texte qui veut devenir ceuvre. Le procès-verbal et le reste des œuvres de la première époque de Le Clézio sont illisibles pour un public de masse; les romans de Modiano ont souvent une fin non conclusive ou décevante et, quand ils proposent la recherche d'un personnage, ils ne se plient pas à la logique du whodunnit (p.e. les détails non significatifs foisonnent). En plus, il est difficile de faire une 
adaptation cinématographique fidèle aux textes des deux Nobels - à l'exception de Mondo, adapté par Tony Gatlif en 1995.

\subsection{Médiatisme des écrivains} est connu pour sa mauvaise élocution et sa difficulté à faire des phrases. Devant les caméras, il se montre « inadéquat, nébuleux et heurté $»^{11}$, et les épitextes des lecteurs lui conseillent même de consulter un ortophoniste $!^{12}$ Il avoue donc se mettre constamment à l'écart du « monde », des interviews, des séances de signatures :

L'écriture est un métier où on est complètement déconnecté, toujours seul. Ce n'est pas un travail collectif comme celui des metteurs en scène de théâtre, par exemple, sans cesse entourés par des gens qui vantent leur génie, sauf, bien sûr, à donner des conférences au PEN Club ou à pratiquer des séances de signatures...

- Vous pliez-vous à l'exercice?

- Non. A mon époque, ça n'existait pas les signatures, alors je continue comme ça, il est normal de ne pas m'y voir. J'imagine qu'un écrivain qui fait des signatures a l'impression d'avoir des lecteurs (Modiano, 2010).

Modiano parle ici, nous le voyons bien, du rite médiatique des dédicaces promotionnelles, non pas des dédicaces d'exemplaires (Nyssen, 1993: 103), qu'il a au contraire toujours pratiquées abondamment... pour être lu, effectivement, et pour sa promotion dans le champ littéraire. Les traces de ces premières dédicaces figurent dans l'ouvrage des Cahiers de l'Herne mentionné ci-dessus.

Nous pouvons également comparer, par rapport au retentissement médiatique, les conférences des Nobels lors des séances de remise du prix. Ces séances sont des rituels proéminents de consécration, donc les deux auteurs avaient intérêt, en principe, à énoncer des sujets particulièrement accessibles au public et acceptables par celui-ci, plutôt que revendicatifs. Mais Le Clézio a choisi de parler des effets de la guerre sur les civils, du lien entre l'alphabétisation et la lutte contre la famine, de l'engagement solidaire de l'écrivain et de l'importance des littératures minoritaires face aux grandes, bien situées dans le champ intellectuel (Le Clézio, 2008). Modiano, de son côté, compare l'écrivain à un acupuncteur, à un somnambule, à un tisserand, à un sismographe ou à un musicien, puis évoque le Paris de l'Occupation, son enfance énigmatique, le thème de la mémoire et de l'oubli, en général (Modiano, 2014a). 
Nobel n'ont pas eu un même accueil positif: paradoxalement, il y a eu de légères réticences pour Modiano. Ainsi, le lendemain de la proclamation des lauréats la presse allemande se réjouit des photos berlinoises posées sur les rayons des étagères de l'écrivain (Radish, 2014). Les Italiens soulignent l'origine italienne de Modiano, ainsi que la déception de ceux qui avaient parié pour Murakami ou Ngugi Wa Thiong ${ }^{14}$. Les Espagnols acceptent sans broncher cet écrivain pour rêveurs (Alemany, 2014). Les Portugais soulignent que parmi les lauréats se trouvait un Portugais, António Lobo Antunes, et que le seul Portugais ayant reçu le prix a été Saramago ${ }^{15}$. En Roumanie une voix s'élève contre la France admirée, mettant l'accent sur la pauvreté thématique et le nombre restreint de lecteurs de l'œuvre modianienne. Mais elle défend pourtant le choix du jury, jugeant qu'il contrecarre l'impérialisme envahissant de la littérature en langue anglaise (Ignatoiu-Sora, 2014). Pour leur part, les Anglo-saxons ont protesté contre un prix qu'ils considèrent trop européen (continental), « the perfect platform from which to counter US cultural hegemony" (Brokes, 2014; Malvern, 2014). Les critiques s'attendaient à voir le prix enfin accordé à Thomas Pynchon ou Philip Roth; de plus, ils mettent en avant l'atout de la grande vente, qui, pour eux, assure la valeur de l'objet vendu: «the snobbish Nobel judges don't like to reward authors who actually sell » (Brockes, 2014). Ils soulignent que Modiano n'est pas connu, qu'il ne faut pas le confondre avec Simenon (un grand, celui-ci), qu'il est ennuyant et que ses romans sont tristes et courts (Crispin 2014). Dans ces appréciations nous retrouvons la différence de critères qui oppose la doxa anglo-saxonne à la continentale ou, plus largement, la lecture ordinaire à la lecture savante.

Pour finir, et une fois revus les éléments de prélégitimation et les stratégies de légitimation, nous pourrions nous demander lequel des deux auteurs a plus de poids dans le champ littéraire. Soulignons d'abord que le style particulier de Modiano a réussi à engendrer un adjectif : "modianesque », qui désigne une situation bizarre, dépaysante, entre la réalité et la fiction. Le style de Le Clézio n'a pas suscité une mobilisation pareille. On parle également de la « petite musique » de Modiano, et non pas de celle de Le Clézio. Mais pour juger du capital symbolique gagné par les deux écrivains, laissons la parole aux lecteurs. Ils s'expriment dans un appel à voter publié dans Le Figaro, répondant à la question « Êtes-vous Modiano ou Le Clézio ? $»^{16}$. De 831 votants, Modiano a obtenu $49 \%$ des voix, tandis que Le Clézio a reçu le reste, $51 \%$. Des pourcentages bien serrés, donc, qui 
prouvent... que Modiano n'a pas bien joué ses cartes : c'est lui qui aurait dû gagner, car Le Figaro est un journal de droite...

\section{BIBLIOGRAPHIE}

ALEMANY, Luis (2014). « Modiano, el Nobel de los soñadores ». El Mundo [on-line] [disponible le 26/01/2016] <URL : http://www.elmundo.es/ cultura/2014/10/09/54366b34ca4741be438b4586.html>. BERL, Emmanuel (1976). Interrogatoire, par Patrick Modiano. Paris : Gallimard. BLANCKEMAN, Bruno (2003). « Modiano, à corps perdu(s) », in Jean-François Louette et Roger-Yves Roche (éds.). Portraits de l'écrivain contemporain. Ceyzerieu : Eds. Champ Vallon, pp. 111-120.

BLANCKEMAN, Bruno (2010). « D'un Nobel l'autre : mutations culturelles et évolutions esthétiques de la littérature narrative en France », Carnets, no spécial automne-hiver, pp. 257-265. [disponible le 26/01/2016] <URL : http://carnets.web.ua.pt/>.

BOURDIEU, Pierre (1992). Les règles de l'art. Genèse et structure du champ littéraire. Paris : Seuil. BROCKES, Emma (2014). « The real scandal of Patrick Modiano's Nobel win is that Philip Roth is a huge loser - again ». The guardian [on-line]. [disponible le 26/01/2016] <URL : http:// www.theguardian.com/commentisfree/emma-brockes-column/2014/oct/09/patrick-modianonobel-prize-literature-prize-philip-roth-loser>.

CHANCEL, Jacques (2014). « Jacques Chancel et les politiques », entretien dans le programme Télématin de T2 [on-line]. [disponible le 26/01/2016] <URL : https://www.youtube.com/watch ? $\mathrm{v}=$ Pioanm5PdZU>.

CORTANZE, Gérard de (1999). J.-M. G. Le Clézio. Le nomade immobile. Paris : Gallimard.

CORTANZE, Gérard de (2009). J.-M. G. Le Clézio. Paris : Gallimard.

COSNARD, Denis (2010). Dans la peau de Patrick Modiano. Paris : Anthème Fayard.

CRISPIN, Jessa (2014). « Patrick Modiano Wins Nobel Prize in Literature, Murakami and Roth Fans Freak Out Right on Schedule ». The Portland Mercury [on-line]. [disponible le 26/01/2016] <URL : http://www.portlandmercury.com/BlogtownPDX/archives/2014/10/09/patrick-modiano-winsnobel-prize-in-literature-murakami-and-roth-fans-freak-out-right-on-schedule>.

DEBRAY, Régis (1991). Cours de médiologie générale. Paris : Gallimard.

GARCIN, Jérôme (2008). « Un Nobel anti-parisien : Le Clézio, l'ami public ». L'Obs [on-line]. [disponible le 26/01/2016] <URL : http://bibliobs.nouvelobs.com/actualites/20081009.BIB2157/ le-clezio-prix-nobel-2008.html>.

GRAVEN, Marcus (2015). « L'écrivain bobo Le Clézio s'est-il converti à l'islam ? ». Riposte laïque [online]. [disponible le 26/01/2016] <URL : http://ripostelaique.com/lecrivain-bobo-le-clezioconverti-a-lislam-symbolise-laffaissement-de-notre-societe.html>. 
IGNĂŢOIU-SORA, Emanuela (2014). « Ce mai înseamnă Premiul Nobel pentru Literatură ? ». Observator cultural [on-line]. [disponible le 26/01/2016] <URL : http://www.observatorcultural.ro/ Ce-mai-inseamna-Premiul-Nobel-pentru-Literatura*articleID_30945-articles_details.html>.

KAPRIÈLIAN, Nelly (2014). « Patrick Modiano, écrire pour réparer le passé ». Les inroks [on-line]. [disponible le 26/01/2016]<URL : http://www.lesinrocks.com/2014/10/26/livres/lartreparation-11529778>.

LE CLÉZIO, Jean-Marie Gustave (1999). J.M.G. Le Clézio. Hier et aujourd'hui. Entretiens avec Pierre Lhoste, Jean-Louis Ezine, Olivier Germain-Thomas. Compacts Radio France.

LE CLÉZIO, Jean-Marie Gustave (2008). Conférence du Prix Nobel [on-line]. [disponible le 25/01/2016] <URL : http://www.nobelprize.org/nobel_prizes/literature/laureates/2008/cleziolecture_fr.html>.

LE CLÉZIO, Jean-Marie Gustave (2012). « La lugubre élucubration de Richard Millet ». L'Obs. [disponible le 25/01/2016] <URL : http://bibliobs.nouvelobs.com/actualites/20120905.OBS1344/ la-lugubre-elucubration-de-richard-millet.html>.

LE CLÉZIO, Jean-Marie Gustave (2015a). « Lettre à ma fille ». Le monde [on-line] [disponible le 26/01/2016] > URL : http://www.lemonde.fr/livres/article/2015/01/14/lettre-a-ma-fille-aulendemain-du-11-janvier-2015-par-jmg-le-clezio_4556225_3260.html>.

LE CLÉZIO, Jean-Marie Gustave (2015b). « Si Marine Le Pen gagne, je rendrai mon passeport français ». Le Point [on-line]. [disponible le 26/01/2016] <URL : http://www.lepoint.fr/culture/leclezio-si-marine-le-pen-gagne-je-rendrai-mon-passeport-francais-07-03-2015-1910965_3.php>. MALVERn, Jack (2014). « Patrick Modiano, the 'Marcel Proust for our time' wins Nobel Prize for Literature ». The Times [on-line]. [disponible le 26/01/2016] <URL: http://www.thetimes.co.uk/ tto/arts/books/article4231957.ece>.

MODIANO, Patrick (1977). Livret de famille. Paris : Gallimard.

MODIANO, Patrick (1982). De si braves garçons. Paris : Gallimard.

MODIANO, Patrick (2010). «Je suis devenu comme un bruit de fond », entretien avec Marianne Payot et Delphine Peras. L'Express [on-line]. [disponible le 26/01/2016] <URL : http:// www.lexpress.fr/culture/livre/patrick-modiano-je-suis-devenu-comme-un-bruit-defond_852718.html>.

MODIANO, Patrick (2014). « Je ne m'attendais pas du tout à remporter le Nobel », entretien avec Nelly Kaprièlian. Les Inroks [on-line]. [disponible le 24/01/2016] <URL : http:// www.lesinrocks.com/2014/10/15/livres/modiano-mon-travail-sorte-retoucheperpetuelle-11529784/>.

MODIANO, Patrick (2014a). Conférence du Prix Nobel [on-line]. [disponible le 25/01/2016] <URL : http://www.nobelprize.org/nobel_prizes/literature/laureates/2014/modiano-lecture_fr.html>. MOLINIÉ, Georges et VIALA, Alain (1993). Approches de la réception. Sémiostylistique et sociopoétique de Le Clézio. Paris : PUF.

NYSSEN, Hubert (1993). Du texte au livre, les avatars du sens. Paris : Nathan.

RADISH, Iris (2014). « Das Geheimnis eines Lebens ». Die Zeit [on-line]. [disponible le 26/01/2016] <URL : http://www.zeit.de/2014/49/patrick-modiano-nobelpreis-literatur>. 
ROBERT, Virgine (2014). « Dans les coulisses du Prix Nobel ». Les Échos [on-line]. [disponible le 26/01/2016] <URL : http://www.lesechos.fr/10/12/2014/LesEchos/21832-045-ECH_dans-lescoulisses-du-prix-nobel.htm>.

VIALA, Alain (1988). « Effets de champ et effets de prisme », Littérature, n 70, pp. 64-71.

\section{NOTES}

1. 15, contre 6 lauréats espagnols et 1 lauréat portugais.

2. Nous ne tenons pas à évaluer ici la pureté ou l'impartialité du jury du Nobel. Lors de l'attribution du prix à Modiano la question a encore été posée. Cf. par exemple, et pour éveiller les soupçons, les mots de Jesper Svenbro, membre du jury, qui avoue des accointances en quelque sorte personnelles avec Modiano: «J'en suis venu assez tardivement à lire Modiano. Je l'ai lu quand j'habitais en France. J'ai commencé avec un livre qui évoquait le bord de la Marne et la Côte d'Azur. Je connaissais. Il y mentionnait une "gourmette" et je savais ce que c'était. Son héros s'appelait Schlemilovitch, ce qui revient à dire "pauvre type". Je comprenais tout cela, et cela a créé pour moi une communication avec l'auteur» (Robert, 2014).

3. http://lereseaumodiano.blogspot.com.es/p/reperes-biographiques.html

4. Ibid.

5. Lors d'un entretien après un déjeuner avec Modiano, la ministre de la Culture Fleur Pellerin a avoué qu'elle n'avait lu aucun de ses livres. Cf. http://www.lefigaro.fr/politique/le-scan/ couacs/2014/10/26/25005-20141026ARTFIG00126-la-ministre-de-la-culture-incapable-de-citerle-nom-d-un-livre-de-patrick-modiano.php [disponible le 26/01/2016].

6. Sachant que, ainsi que l'affirme Viala, les écrivains sont guidés en une certaine mesure, même dans le choix des sujets, par les exigences du champ littéraire : « Le choix des sujets et des façons de les traiter apparaît ainsi comme, non pas un hasard ou une liberté pure de l'écrivain, mais bien comme une liberté relative, dont la marge de manœuvre équivaut à ce que le prisme du champ des prises de position possibles permet » (Viala, $1988: 69$ ).

7. Cortanze, 1999 : 154-155. Il fut muté au Mexique et obligé de finir là-bas son service militaire.

8. La prise de parole politique dans les médias a commencé en septembre 2012 : après le massacre de l'île d'Utoya, en Norvège, et la défense de l'assassin par Richad Millet dans un pamphlet, Le Clézio fait paraître un article pour dénoncer les absurdités du racisme, de la xénophobie et des idées monoidentitaires (Le Clézio, 2012).

9. Toutefois Modiano avait expliqué à cette même journaliste : «Comme j'écris des ouvrages de fiction, je ne peux pas échapper à mon époque. Même si on décide de vivre dans une tour d'ivoire, tout ce qu'on peut faire est forcément traversé par l'air du temps. On n'y échappe pas, on la ressent, comme un sismographe, et même si mes livres n'en rendent pas compte explicitement, le temps dans lequel j'écris mes livres s'y reflète » (Modiano, 2014).

10. http://www.dailymotion.com/video/x8difp_jean-marie-le-clezio-prix-nobel-de_creation [disponible le 26/01/2016].

11. http://www.lepoint.fr/culture/modiano-portrait-du-romancier-eninterviewe-10-10-2014-1871153_3.php

http://www.lepoint.fr/culture/modiano-portrait-du-romancier-eninterviewe-10-10-2014-1871153_3.php [disponible le 26/01/2016].

13. http://www.leparisien.fr/loisirs-et-spectacles/patrick-modiano-decroche-le-prix-nobel-delitterature-09-10-2014-4199995.php [disponible le 26/01/2016].

14. http://www.corriere.it/cultura/14_ottobre_09/nobel-la-letteratura-patrick-modianod8e9e7a4-4f9e-11e4-8d47-25ae81880896.shtml?refresh_ce-cp [disponible le 26/01/2016]. 
15. http://www.dn.pt/artes/livros/interior/um-portugues-entre-os-110-laureados-e-outrascuriosidades-4163958.html [disponible le 26/01/2016].

16. http://www.lefigaro.fr/culture/2010/07/19/03004-20100719QCMWWW00405-tes-vousmodiano-ou-le-clezio.php [disponible le 26/01/2016].

\section{RÉSUMÉS}

Cet article compare la situation des prix Nobels Le Clézio et Modiano dans le champ intellectuel européen, examinant d'abord le capital familial et social de départ, ensuite les sujets abordés dans les œuvres des deux écrivains, en tant qu'accélérateurs ou décélérateurs du cursus honorum, et enfin les stratégies promotionnelles et médiatiques de la réussite.

This article compares the situation of two French Nobels, Le Clézio and Modiano, in the European literary field. The author deals first with the family and social capital, then with the topics treated in the books of both writers, as they slow or accelerate the path in the cursus honorum, finally with the promotional and media strategies needed to succeed.

\section{INDEX}

Mots-clés : Le Clézio (J.-M. G.), Modiano (Patrick), Prix Nobel de littérature, champ littéraire, européen

Keywords : Le Clézio (J.-M. G.), Modiano (Patrick), Nobel Prize in Literature, literary field, European

\section{AUTEUR}

\section{PILAR ANDRADE BOUÉ}

Un. Complutense de Madrid pandrade[at]ucm.es 